\title{
A Community-based Lifestyle Education Program Addressing Non-communicable Diseases in Low-literacy Areas of the South Pacific: A Pilot Control Cohort Study
}

\author{
Lillian Kent*, Pia Reierson, Darren Morton, Kesa Vasutoga, Paul Rankin
}

\begin{abstract}
Lifestyle interventions can effectively reduce chronic disease risk factors. This study examined the effectiveness of an established lifestyle intervention contextualized for low-literacy communities in Fiji. Ninety-six adults from four villages, with waist circumference (WC) indicative of risk of chronic disease, were randomly selected to an intervention or control group. Process evaluation indicated one intervention and one control village fulfilled the study protocol. There were no differences between intervention and control for body mass index BMI $(P=0.696)$, WC $(P=0.662)$, total cholesterol (TC) $(P=0.386)$, and TC:high-density lipoprotein (HDL) ratio $(P=0.485)$. The intervention village achieved greater reductions than the control village at 30 and 90 days for systolic blood pressure (30 days: $-11.1 \%$ vs. $-2.5 \%, P=0.006 ; 90$ days: $-14.5 \%$ vs. $-6.7 \%, P=0.019)$; pulse rate ( 30 days: $-7.0 \%$ vs. $-1.1 \%, P=0.866 ; 90$ days: $-7.1 \%$ vs. $4.3 \%, P=0.027$ ), and HDL ( 30 days: $-13.9 \%$ vs. $1.7 \%$, $P=0.206$; 90 days: $-18.9 \%$ vs. $2.2 \%, P=0.001)$; at 90 days only for diastolic blood pressure $(-14.4 \%$ vs. $-0.2 \%, P=0.010)$; at 30 days only for low-density lipoprotein $(-11.6 \%$ vs. $8.0 \%, P=0.009)$; and fasting plasma glucose $(-10.2 \%$ vs. $4.3 \%, P=0.032)$. However, for triglycerides, the control achieved greater reductions than the intervention village at 30 days $(35.4 \%$ vs. $-12.3 \%, P=0.008$; marginal at 90 days $16.4 \%$ vs. $-23.5 \%$, $P=0.054)$. This study provides preliminary evidence of the feasibility and potential effectiveness of the intervention to lower several risk factors for chronic disease over 30 days in rural settings in Fiji and supports consideration of larger studies.
\end{abstract}

Keywords: Complete Health Improvement Program, Lifestyle education program, Low literacy, Non-communicable disease, Regenerated Freirean Literacy through Empowering Community Techniques, South Pacific

Asian Pac. J. Health Sci., (2020); DOI: 10.21276/apjhs.2020.7.3.2

\section{INTRODUCTION}

Globally, cardiovascular diseases (CVDs), cancer, chronic respiratory diseases, and diabetes constitute the four main noncommunicable diseases (NCDs). Of these, diabetes is increasing rapidly, with 422 million people in the world living with the condition in 2014. ${ }^{[1]}$ While NCDs are responsible for 41 million deaths each year, equivalent to $71 \%$ of all global deaths, ${ }^{[2]}$ these four groups of conditions account for $80 \%$ of premature deaths for individuals between 30 and 69 years. ${ }^{[3]}$ Diabetes alone was responsible for an estimated 1.6 million deaths in 2016. ${ }^{[1]}$

Overweight and obesity, CVD, and diabetes have increased substantially in low-middle-income countries (LMICs), ${ }_{{ }^{[4]}}$ which now bare $85 \%$ of the global burden of premature deaths from NCDs. ${ }^{[3]}$ Furthermore, $80 \%$ of people with diabetes live in LMICs, with the greatest proportion of these (37\%) in the Western Pacific, which includes the South Pacific. ${ }^{[5]}$ Seven of the top 10 countries in the world with the highest prevalence of diabetes are South Pacific Islands..$^{[5]}$ LMIC countries now face a double burden of disease from communicable and NCDs, ${ }^{[2]}$ which has resulted in significant social disadvantage and economic burden on individuals, families, and society at large ${ }^{[4]}$ with economic losses of US\$7 trillion projected by 2030 , resulting in millions of people trapped in poverty. ${ }^{[6]}$

Unhealthy lifestyle is one of the major risk factors of NCDs. ${ }^{[3]}$ Indeed, in Pacific Island countries, the pursuit of better opportunities has raised expectations and together with declining subsistence farming has increased the demand for imported foods; rice and wheat flour are replacing locally grown carbohydrates, with the consumption of poor-quality protein such as mutton flaps, turkey tails, and Spam. ${ }^{[7]}$ Lifestyle interventions that promote shifts in unhealthy eating patterns have been shown to be efficacious for the primary, secondary, and early tertiary
Lifestyle and Health Research Centre, Avondale University College, 582 Freemans, Cooranbong, NSW, Australia

Corresponding Author: Lillian Kent, Lifestyle and Health Research Centre, Avondale University College, 582 Freemans, Cooranbong, NSW, Australia. Email: lillian.kent@avondale.edu.au

How to cite this article: Kent L, Reierson P, Morton D, Vasutoga $K$, Rankin P. A Community-based Lifestyle Education Program Addressing Non-communicable Diseases in Low-literacy Areas of the South Pacific: A Pilot Control Cohort Study. Asian Pac. J. Health Sci., 2020; 7(3):5-10

Source of support: Nil

Conflicts of interest: None

Received: 10/04/2020 Revised: 26/05/2020 Accepted: 15/06/2020

prevention of NCDs. ${ }^{[8,9]}$ However, there is a paucity of evidence regarding effective community-based lifestyle interventions in rural areas of LMICs. ${ }^{[10]}$

The Complete Health Improvement Program (CHIP) is a community-based lifestyle intervention that has demonstrated significant benefits for the management of CVD, ${ }^{[11-13]}$ type 2 diabetes mellitus, ${ }^{[12]}$ and depression, ${ }^{[14,15]}$ using both randomized controlled trial and pre-test/post-test cohort study designs. ${ }^{[16]}$ However, the program was originally developed for middle to high socioeconomic status groups in the United States. Studies from other high-income countries, such as Australia/New Zealand and Canada, ${ }^{[17,18]}$ have also shown high levels of effectiveness, despite the inherent cultural differences.

There have been no published studies of the effectiveness of the CHIP intervention in the Pacific Islands to date. Furthermore, the program in its current format is less suited to the rural and most marginalized parts of the Pacific Islands as: English literacy 
is required, the content is not culturally identifiable, ingredients for suggested recipes are not locally available, and the program requires technical equipment that is not commonly accessible such as video projection. As a result, a contextualized version of the CHIP intervention using an adult learning and social change approach known as Regenerated Freirean Literacy through Empowering Community Techniques (REFLECT) was developed. ${ }^{[19]}$ REFLECT, developed in the 1990s for LMICs, initially focused on linking adult literacy to empowerment, but in more recent times, the approach has been used in various contexts, to enact social change, financial security, literacy/numeracy, and health and well-being. ${ }^{[20,21]}$

Hence, it was rationalized that the REFLECT approach might be appropriate for delivering a program specifically targeting NCDs. The purpose of this pilot study was to examine the efficacy of the adapted version of the CHIP intervention, called "Live More Abundantly (LMA)," to improve health and well-being in Fiji.

\section{Methods/Design}

\section{Study Design}

LMA was delivered to 24 men and 24 women in each of two rural/ semi-rural villages and within a $2 \mathrm{~h}$ drive from Suva, Fiji between August 2014 and February 2015. Two control villages also comprised the same number of men and women and met the same criteria for rurality and proximity from Suva. Villages themselves were separated by $1 \mathrm{~h}$ drive to minimize contamination between intervention and control groups. Villages meeting the selection criteria were randomly selected into intervention or control by the lead researcher, located in Australia, who was not familiar with the villages. The study was approved by the Human Research Ethics Committee (name blinded) in New South Wales, Australia (ID: 2014/03) on March 18, 2014.

\section{Power Calculation}

Data from CHIP interventions conducted in Australia and New Zealand $^{[17]}$ were used for the power analyses. Power calculations on all biometrics measured in the present study found that triglycerides (TG) would require the greatest sample size to achieve statistical significance. Using an estimated mean baseline triglyceride level of $1.15 \mathrm{mmol} / \mathrm{L}$ and a standard deviation of $0.33 \mathrm{mmol} / \mathrm{L}$, power calculations showed that we required 87 participants to achieve a $5 \%$ level of significance with $80 \%$ power. Allowing for $10 \%$ loss to follow up at 6 months, a total of 96 participants were recruited, constituting two intervention villages each with 24 participants and two control villages each with 24 participants.

\section{Village and Participant Recruitment}

The villages where chief's/community elders were supportive and committed to the study were recruited for subsequent randomization into intervention or control sites. ${ }^{[22]}$ To be eligible, the villages were also required to have relatively low literacy rate/ education level, be committed to participating if allocated to intervention or control and have at least $40 \%$ overweight residents by visual assessment. Figure 1 shows the sequence for allocation of sites to treatment and control conditions.

Following randomization, the project supervisor and a health professional (HP - doctor or nurse) recruited eligible participants subsequent to an information session delivered at a chief-initiated village meeting. Participant eligibility criteria included: 18 years of age or older; live permanently in the village (for the duration of the program); have a waist circumference (WC) of $\geq 92 \mathrm{~cm}$ for men and $\geq 80 \mathrm{~cm}$ for women (as levels at and above these are indicative of risk of NCD ${ }^{[22]}$ and be able to participate in the intervention. To this extent, participants were included if they agreed to engage in the intervention and were able to provide their own meals. Participant exclusion criteria included: Unstable angina; myocardial infarction within the previous 12 months of the study; coronary by-pass surgery within the previous 12 months of the study; and other medical contraindications for dietary change or increased physical activity, as determined by the HP. The HP also regularly monitored the health of the participants for the duration of the trial, although the nature of the intervention did not expect to produce harm. Informed consent was obtained by the participant signing/ making their mark on the consent form or giving the appointed HP for the study verbal consent to sign on their behalf in the presence of a witness (in case of literacy constraints). No payments were requested of participants and the participants did not receive any financial remuneration for their involvement.

\section{Village Facilitators}

One male and one female program facilitator, recommended by the community leaders of the respective intervention villages, received 2 weeks of training. Sessions covered the principles of the CHIP intervention and the REFLECT methodology ${ }^{[20]}$ as well as the development of facilitation skills and the research methods used in the study. Eligibility criteria included: Completed secondary education, English fluency, be respected in the village, a non-smoker, and have an interest in health with a desire to positively impact the health of their village. The supervisor was responsible for follow-up, mentoring the village facilitator, and monitoring the program and research.

\section{Intervention}

Participants received 90 days of the contextualized 18-session LMA Program that is based on and follow the order of the 18-session "Westernized" CHIP intervention. ${ }^{[16]}$ The program encourages and supports the participants to move toward a low-fat, plant-based diet ad libitum, with emphasis on the whole-foods consumption of grains, legumes, fruits, and vegetables, to engage in $30 \mathrm{~min}$ of moderate-intensity physical activity daily and practice stress management techniques. Consumption of a low-fat plant-based diet is associated with reduced risk of obesity and chronic diseases, including CVD and diabetes. ${ }^{[23]}$ Session topics included: "The Rise and Rise of Chronic Disease;" "Lifestyle is the Best Medicine;" "The Common Denominator of Chronic Disease;" "The Optimal Lifestyle;" "Eat More, Weigh Less;" "Fiber, Your New Best Friend;" "Disarming Diabetes;" "The Heart of the Matter - Heart Health;" "Controlling blood pressure and discovering protein;" "'Bone Health Essentials;" "Cancer Prevention;" "How to grow a family garden. What to grow and when?" "Become what you believe and your DNA is not your destiny;" "Practicing forgiveness;" "Re-engineering your environment;" "Stress-relieving strategies;" "Fix how you feel;" and "From Surviving to Thriving." Each session typically involved meeting in the "REFLECTCircle" where all participants had the opportunity to participate and feel included. A typical session includes a welcome, a participatory methods exercise to draw the 


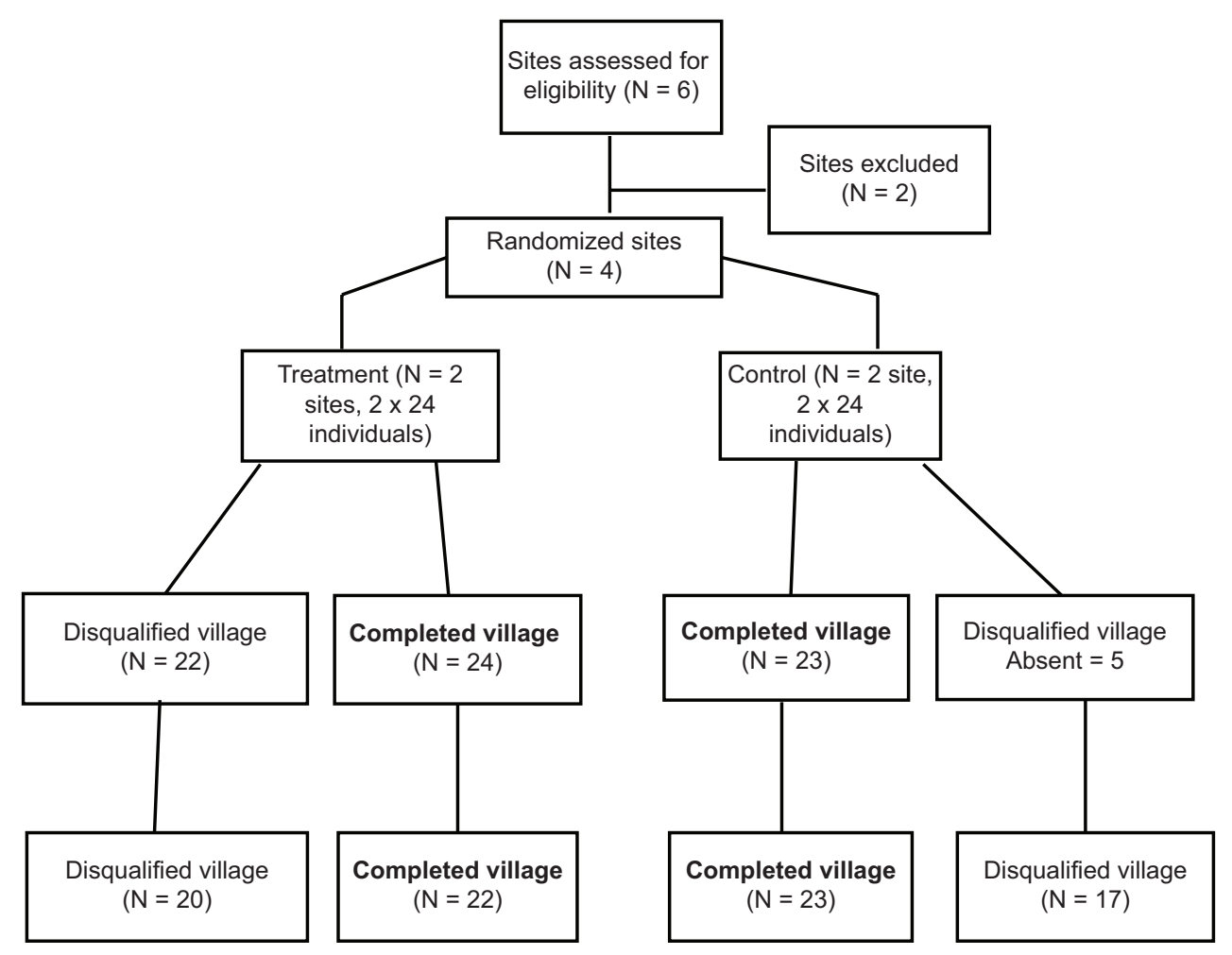

Figure 1: Consort of flow

individual's knowledge/experiences of the topic for that session, for example, map, calendar, matrix, and role play, a trigger picture then introduces the topic for that session, the researched content is discussed by the facilitator and participants work in pairs or small groups to formulate an action plan to decipher how the change can be incorporated into their lifestyle and closes with a conclusion. ${ }^{[22]}$

Participants met 3 times a week in the first 30 days, then once a week until the end of the intervention. These sessions were translated and delivered in the local vernacular.

Participants were considered to have completed the program if they attended 14 of the 18 sessions and biometric testing at alltime points. Participants celebrated their achievements in a group feedback session with the distribution of graduation certificates, followed by a LMA friendly feast.

\section{Control Villages}

In each of the control villages, printed health education material developed by the local Ministry of Health $(\mathrm{MoH})$ was presented at the first health screening. At each subsequent assessment point, the HP invited the individual to ask any health-related questions from the health literature provided at baseline.

\section{Outcome Measures and Measurement Points}

Data on biometrics (height, weight to calculate body mass index [BMI]; WC and blood pressure), $12 \mathrm{~h}$ fasting blood measures, and health behavior were collected on individuals in the intervention and control villages at program entry (baseline), 30 days, and 90 days by a team of HPs. The blood samples were tested for total cholesterol (TC), low-density lipoprotein (LDL), high-density lipoprotein (HDL), TG, and fasting plasma glucose (FPG) levels using whole blood from finger prick and analyzed on site using the CardioChek PA portable blood analyzer. Health-related behaviors were collected at each time point using a lifestyle questionnaire, which included smoking, alcohol, and kava usage; dietary intake; physical activity (as measured by a pedometer provided to the participant); and subjective well-being. Personal and family health history, including medication usage, was collected at program entry only.

During the process evaluation, we discovered that one of the facilitators in one of the intervention villages (Gusuisavu) emigrated to another village soon after the program commenced but was not replaced by the village leaders. The remaining facilitator did not have the status to draw the community together and so meetings were not regular. Even when meetings were held, attendance was poor and the entire program was not completed. In one of the control villages (Navuso), we learned that this was contaminated by the health team providing additional health advice to the participants during the baseline health assessments, above that provided in the $\mathrm{MoH}$ leaflets. Hence, only the findings for one intervention (Naganivatu) and one control (Sawani) village are presented.

\section{Statistical Methods}

The (biomedical) data were analyzed by the lead researcher in Australia, using $\mathrm{IBM}^{\mathrm{TM}}$ Statistics (version 21). Continuous data were expressed as number, mean, and SD. Independent samples $t$-tests for continuous variables were used to examine baseline differences between participants in the intervention and control groups. The extent of changes (percentage and mean with $95 \%$ confidence intervals) from baseline, 30 days, and 90 days was 
assessed using analysis of variance (repeated measures) for the control and intervention villages. Where significant interaction between the villages was found, one-way repeated measures ANOVA was then conducted on the relevant biometric. Where differences were found between intervention and control villages, analysis of covariance with controlling for the relevant baseline variable was used to determine changes at each time point. For all analyses, results are statistically significant at $P<0.05$.

\section{Results}

\section{Recruitment to the Trial}

Recruitment to the trial occurred between August 2014 and end of September 2014. Figure 1 shows the participant flow through the study. Six villages were approached, informed of the study objectives and asked their willingness to participate. Four villages met the selection criteria.

\section{Baseline}

There was no difference in the mean age of the intervention and control groups $(49.0 \pm 12.6$ years vs. $46.2 \pm 16.6$ years, $\mathrm{t}(73)=-0.827, P=0.411)$. Both groups were representative of an at-risk population with mean BMI in the "obese" category and elevated systolic blood pressure (SBP), TC, LDL, and TG, and low HDL [Table 1]. Furthermore, no significant differences were found for BMI $(\mathrm{t}(79)=-0.895, P=0.373), \mathrm{WC}(\mathrm{t}(78)=-0.504, P=0.615)$, SBP $(\mathrm{t}(79)=0.822, P=0.413)$, diastolic blood pressure (DBP) $(\mathrm{t}(79)=-0.598, P=0.552)$, pulse rate $(\mathrm{t}(79)=-0.078, P=0.938)$, FPG $(\mathrm{t}(77)=-0.638, P=0.525), \mathrm{TC}(\mathrm{t}(77)=-0.309, P=0.758), \mathrm{TG}$ $(\mathrm{t}(76)=-1.857, P=0.067), \mathrm{LDL}(\mathrm{t}(76)=-0.094, P=0.926)$, and TC:HDL ratio $(\mathrm{t}(77)=-1.807, P=0.075)$ [Table 1]. However, the intervention group had higher mean HDL than the control group $(\mathrm{t}(77)=2.134, P=0.036)$ [Table 1].

\section{Follow-up}

Biometric data were collected for $94 \%$ of participants at 30 days and $86 \%$ at 90 days. At baseline, one participant from the intervention village did not fast at baseline and 90 days, so fasting glucose and lipids were not measured. For the control village, one participant did not attend the assessment at 30 days but returned at 90 days. For all other participants, all outcome data were collected.

Statistically significant reductions were observed in the 30 days means for all risk factors, except for pulse and TC in the intervention village. Conversely, TG significantly increased about $35 \%$. The decrease in all risk factors in this village was significantly greater at 90 days, except WC [Table 1]. However, the large increase in TG observed at 30 days attenuated at 90 days. By comparison, participants from the control village only achieved sporadic reductions [Table 1].

There were no differences between intervention and control for BMI $(F(1,43)=0.155, P=0.696), W C(F(1,42)=0.194, P=0.662)$, TC $(F(1,42)=0.766, P=0.386)$, and TC:HDL ratio $(F(1,42)=0.497$, $P=0.485)$. Significant interaction was found between the villages for the other biometrics. The intervention village achieved greater changes than the control village for $\operatorname{SBP}(F(2,20)=38.82$, $P=<0.001$, partial eta-squared $=0.795$ vs. $F(2,21)=4.97, P=0.017$, partial eta-squared $=0.321), \operatorname{DBP}(F(2,20)=11.24, P=0.001$,

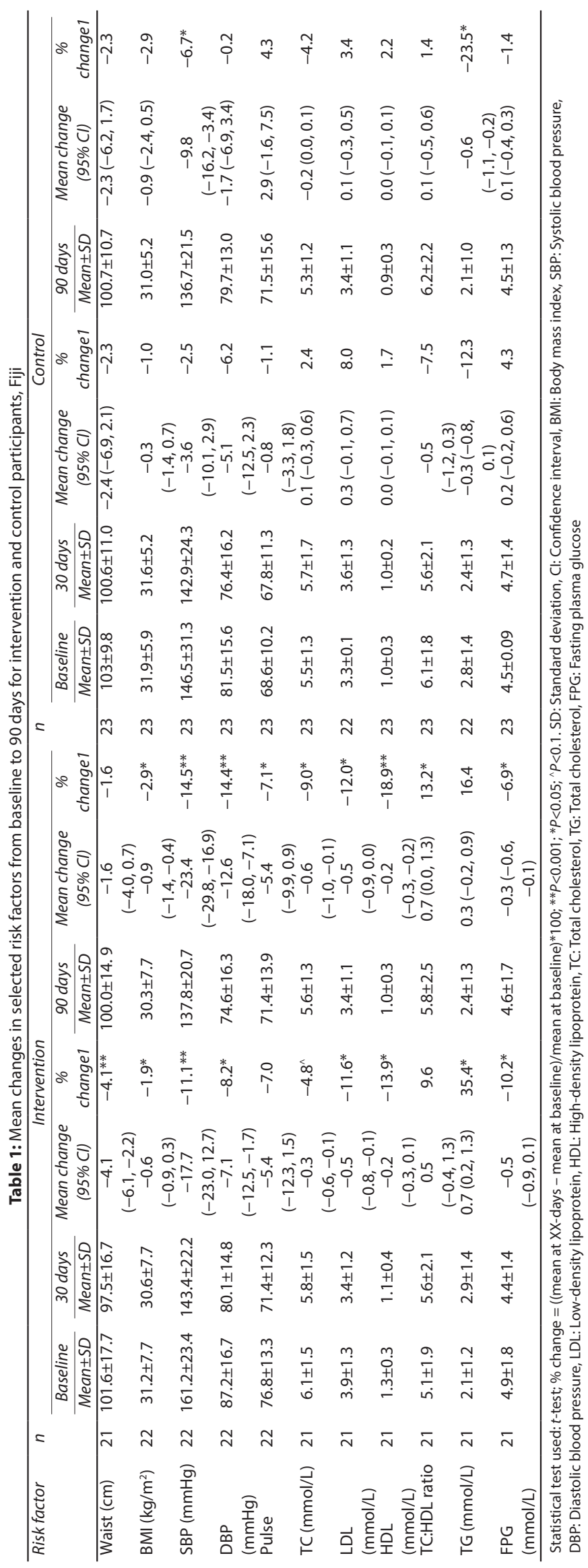


partial eta-squared $=0.529$ vs. $F(2,21)=1.014, P=0.380)$, FPG $(F(2,19)=5.157,0=0.016$, partial eta-squared $=0.352$ vs. $F(2,21)$ $=1.408, P=0.267), \mathrm{HDL}(\mathrm{F}(2,19)=44.273, P<0.001$, partial etasquared $=0.823$ vs. $\mathrm{F}(2,21)=0.318, P=0.731), \mathrm{TG}(\mathrm{F}(2.19)=8.379, P$ $=0.002$, partial eta-squared $=0.469$ vs. $F(1,21)=0.380, P=0.039$, partial eta-squared $=0.266)$, and almost significant for $\operatorname{LDL}(F(2,19)$ $=3.409, P=0.054$, partial eta-squared $=0.264$ vs. $\mathrm{F}(2,21)=1.154$, $P=0.334)$ and pulse rate $(F(2,20)=3.002, P=0.072$, partial etasquared $=0.321$ vs. $F(2,21)=1.634, P=0.219)$.

These changes were found at both time points for SBP (30 days: $\mathrm{F}(1,42)=8.479, P=0.006 ; 90$ days: $\mathrm{F}(1,42)=5.976 P=0.019)$, pulse rate $(30$ days: $F(1,42)=0.029 P=0.866 ; 90$ days: $F(1.42)=5.228, P=$ 0.027 ), and HDL (30 days: $F(1,41)=1.648, P=0.206 ; 90$ days: $F(1,41)$ $=13.748, P=0.001)$. Greater reductions were achieved by the intervention village at 90 days only for $\mathrm{DBP}(30$ days: $\mathrm{F}(1,42)=0.023$, $P=0.879 ; 90$ days: $F(1,42)=7.319, P=0.010) ; 30$ days only for $L D L$ (30 days: $F(1,41)=4.952, P=0.032 ; 90$ days: $F(1,41)=1.422, P=$ $0.240)$ and FPG (30 days: $F(1,41)=5.723, P=0.021 ; 90$ days: $F(1,41)$ $=1.908, P=0.301)$. However, for TG, the control village achieved greater reductions than the intervention village (30 days: $F(1,41)$ $=7.663, P=0.008$; marginal at 90 days $F(1,41)=3.929, P=0.054)$.

\section{Discussion}

This pilot study provides preliminary evidence of the feasibility and potential effectiveness of the modified and contextualized version of CHIP to reduce risk factors for chronic disease in low literacy and resource poor areas, such as semi-rural communities in Fiji. Specifically, the reductions in blood pressure and pulse rate continued to improve to 90 days. Furthermore, greater reductions were seen at 30 days for LDL and FPG, but these were tempered by 90 days. That these potential outcomes can be achieved with a volunteer-delivered, community-based intervention is significant, given the burgeoning rise in chronic disease in the South Pacific. However, larger scale studies are needed to confirm these findings.

Nonetheless, although we only had a small number of participants, a comparison of the risk factor reductions in this study with those recently reported in other countries showed that the outcomes in this pilot study were comparable to those of standard CHIP programs elsewhere, ${ }^{[1,17,18,24]}$ and for some biometrics, changes were even greater. Previously, we have found that people who enter the program with the worst biometrics tend to achieve greater change and improvement. ${ }^{[11,17,18]}$ Comparison of baseline biometrics from these studies would indicate that baseline levels at which participants enter the program do affect the differences in change for the various biometrics.

There were several anecdotal reports in the present study of participants' having their medications (e.g., hypertensive, hypercholesterolemia, and hyperglycemic) decreased or even ceased by their personal doctor during the intervention. While this is a desirable outcome, a reduction in medication usage may have caused the results presented in this report to be understated. It is a limitation of this study that medication changes were not recorded except at baseline and this will be included in future studies.

These large changes, as shown by the partial eta-squared, observed with the LMA intervention are likely a result of the program's emphasis on a whole-food, plant-based eating pattern, which is largely free from exogenous cholesterol, low in saturated fat, and high in fiber. The substantial reduction in serum LDL cholesterol ( $L D L-C)$, leading to a decreased need for reverse cholesterol transport, may also explain the acute reduction in
HDL-C seen in this study and others that advocated a plant-based eating pattern. ${ }^{[25-27]}$

\section{Strengths and Limitations}

This is the only study assessing a contextualized version of the standard CHIP program in the South Pacific. The strengths are the consistent use of messages from the evidence-based standard CHIP program, the use of the globally recognized REFLECT methodology, and the measurement of biometrics by HPs using consistent tools across all time points. A unique element of the study was the use of volunteers to administer a lifestyle intervention. The comparability of the outcomes in this study with those of larger CHIP studies is also a strength of the study and suggests the efficacy of the LMA intervention.

There are a number of limitations in this study. Despite the comprehensive list of selection criteria, one intervention village did not complete the intervention as the principal facilitator migrated leaving the assistant facilitator who was not as respected within the village as originally thought. This halved the number of participants and therefore lowered the power required to find statistical significance in outcomes. On the other hand, the large effect sizes observed in the successfully completed intervention village further indicate the efficacy of the intervention to address some chronic disease risk factors. For broader delivery of the program, recruitment of facilitators needs to be more stringent with the conduct of interviews to ascertain attitudes toward health, support by village members, and experience in leadership activities. Utilizing passionate volunteers to facilitate lifestyle interventions, as they possess motivational properties to incite their peers to action, represents a potentially powerful and costeffective mode for the developing world context. ${ }^{[28]}$

It is not clear why triglyceride levels increased following the intervention when in the other international sites, TG decreased substantially. We found that there was some ambiguity in the message to eat more fruit, with a perception among the participants that this includes fruit juice. High sugar intake has been shown to raise TG, and fruit juice is high in sugar. ${ }^{[29]} \mathrm{A}$ distinction between whole fruit consumption and other processed forms will be included in the program content going forward.

Another limitation of the study was the short duration of the follow-up post-intervention. A small New Zealand study found longer term benefits of the standard CHIP program, on average 4 years after completion of the intervention. ${ }^{[30]}$ Given the comparability of the outcomes in this study with standard CHIP programs from various countries, we would expect similar longterm benefits following completion of the LMA intervention. Nevertheless, longer-term studies are required to assess recidivism following completion of the LMA intervention.

A further limitation of the study is that no consideration was made of population approaches to health promotion through the media and changes/restriction on marketing and price of food, beverages, tobacco, etc. The authors do not believe that these issues are significant in this context as the program was developed for illiterate, marginalized, and remote communities in LMIC.

\section{Conclusions}

This is the first lifestyle intervention using the REFLECT approach to target NCDs in the South Pacific. It provides preliminary evidence of the feasibility and potential effectiveness of the intervention to 
lower several risk factors for chronic disease over 30 days in rural settings in Fiji. This study supports consideration of larger studies to test the interventions efficacy with the following modifications to ensure success: More careful selection of facilitators, clarity of protocol to HPs in conducting health assessment, and clarification of messages in sessions about what constitutes healthy lifestyle behaviors.

\section{ACKnOWLedgments}

We are thankful to the funders of this trial, ADRA Australia.

\section{References}

1. WHO. Diabetes; 2018. Available from: https://www.who.int/newsroom/fact-sheets/detail/diabetes. [Last accessed on 2019 Sep 04].

2. WHO. World Health Statistics 2014. In: Media Centre; 2014. Available from: http://www.who.int/mediacentre/news/releases/2014/worldhealth-statistics-2014/en. [Last accessed on 2014 Aug 08].

3. WHO. Non Communciable Diseases; 2018. Available from: https:// www.who.int/news-room/fact-sheets/detail/noncommunicablediseases. [Last Accessed 2019 Sep 04].

4. Omran AR. The epidemiologic transition. A theory of the epidemiology of population change. Milbank Mem Fund Q 1971;49:509-38.

5. International Diabetes Federation. 2017. IDF Diabetes Atlas. $8^{\text {th }}$ ed. Available from: http://www.diabetesatlas.org/across-the-globe.html. [Last accessed on 2018 Oct 25].

6. WHO. Global Action Plan for the Prevention and Control of Noncommunicable Diseases 2013-2020. In: Noncommunicable Diseases and Mental Health; 2013. Available from: http://www.who. int/nmh/events/ncd_action_plan/en. [Last accessed on 2014 Sep 11].

7. Russell L. Poverty, Climate Change and Health in Pacific Island Countries. Issues to Consider in Discussion, Debate and Policy Development; 2011. Available from: http://www.hdl.handle. net/2123/9202. [Last accessed on 2019 Sep 04].

8. Ornish D, Brown SE, Scherwitz LW, Billings JH, Armstrong WT, Ports TA, et al. Can lifestyle changes reverse coronary heart disease? The lifestyle heart trial. Lancet 1990;336:129-33.

9. Esselstyn CB Jr., Ellis SG, Medendorp SV, Crowe TD. A strategy to arrest and reverse coronary artery disease: A 5-year longitudinal study of a single physician's practice. J Fam Pract 1995;41:560-8.

10. van de Vijver S, Oti S, Addo J, de Graft-Aikins A, Agyemang C. Review of community-based interventions for prevention of cardiovascular disease in low and middle-income countries. Ethnic Health 2012;17:651-76.

11. Rankin P, Morton DP, Diehl H, Gobble J, Morey P, Chang E. Effectiveness of a volunteer-delivered lifestyle modification program for reducing cardiovascular disease risk factors. Am J Cardiol 2012;109:82-6.

12. Diehl HA. Coronary risk reduction through intensive communitybased lifestyle intervention: The CHIP experience. Am J Cardiol 1998;82:83T-7.

13. Englert HS, Diehl HA, Greenlaw RL, Willich SN, Aldana S. The effect of a community-based coronary risk reduction: The Rockford CHIP. Prev
Med 2007;44:513-9.

14. Merrill RM, Taylor P, Aldana SG. Coronary health improvement project (CHIP) is associated with improved nutrient intake and decreased depression. Nutrition 2008;24:314-21.

15. Thieszen CL, Merrill RM, Aldana SG, Diehl HA, Mahoney ML, Greenlaw $\mathrm{RL}$, et al. The coronary health improvement project (CHIP) for lowering weight and improving psychosocial health. Psychol Rep 2011;109:338-52.

16. Morton DP, Rankin PM, Kent LM, Dysinger W. The complete health improvement program (CHIP): History, evaluation, and outcomes. Am J Lifestyle Med 2014;10:64-73.

17. Morton DP, Rankin P, Morey P, Kent L, Hurlow T, Chang E, et al. The effectiveness of the complete health improvement program (CHIP) in Australasia for reducing selected chronic disease risk factors: A feasibility study. N Z Med J 2013;126:43-54.

18. Morton DP, Rankin P, Kent L, Sokolies R, Dysinger W, Gobble J, et al. The effectiveness of the complete health improvement program (CHIP) in Canada for reducing selected chronic disease risk factors. Can J Diet Pract Res 2014;75:72-7.

19. Reflect. Available from: http://www.reflect-action.org. [Last accessed on 2014 Mar 03].

20. Sempere, K. Counting Seeds for Change; A Framework for Implementing, Monitoring and Evaluating Reflect. Johannesburg; 2009. Available from: http://www.reflect-action.org/sites/default/ files/u5/Reflect\%20Evaluation\%20Framework\%20-\%20English.pdf. [Last accessed on 2014 Mar 03].

21. Howie B. Participatory Evaluation of ALIVE. United States: ADRA Australia; 2014.

22. Kent LM, Reierson P, Morton DP. Live more: Study protocol for a community-based lifestyle education program addressing noncommunicable diseases in low-literacy areas of the South Pacific. BMC Public Health 2015;15:1221-8.

23. Tuso PJ. Nutritional update for physicians: Plant-based diets. Perm J 2013;17:61-6.

24. Kent LM, Morton DP, Mañez JT, Yabres GD, Muya AB, Rankin PM, et al. The complete health improvement program (CHIP) and reduction of chronic disease risk factors in the Philippines. Asian Pac J Health Sci 2015;2:67-75.

25. Kent L, Morton D, Rankin P, Ward E, Grant R, Gobble J, et al. The effect of a low-fat, plant-based lifestyle intervention (CHIP) on serum HDL concentrations and the implications for metabolic syndrome status-a cohort study. Nutr Metab 2013;10:58.

26. Ferdowsian HR, Barnard ND. Effects of plant-based diets on plasma lipids. Am J Cardiol 2009;104:947-56.

27. Ornish D. Serum lipids after a low-fat diet. JAMA 1998;279:1345-6.

28. Kong BW. Community-based hypertension control programs that work. J Health Care Poor Underserved 1997;8:409-15.

29. Hudgins LC, Seidman CE, Diakun J, Hirsch J. Human fatty acid synthesis is reduced after the substitution of dietary starch for sugar. Am J Clin Nutr 1998;67:631-9.

30. Kent L, Morton D, Hurlow T, Rankin P, Hanna A, Diehl H. Long-term effectiveness of the community-based complete health improvement program (CHIP) lifestyle intervention: A cohort study. BMJ Open 2013;3:e003751. 